\title{
PiRNA MW557525 Promotes the Vitality and Pluripotency of Piwil2-iCSCs by Regulating NOP56.
}

\section{Zhaoxia Zhang}

Chongqing Medical University

Zhang Wang ( $\sim 273399430 @ q q . c o m$ )

Chongqing Medical University

Xiaojun Tan

Chongqing Medical University

Liming Jin

Chongqing Medical University

Zhaoying Wang

Chongqing Medical University

\section{Lianju Shen}

Chongqing Medical University Affiliated Children's Hospital

Chunlan Long

Chongqing Medical University Affiliated Children's Hospital

Guanghui Wei

Chongqing Medical University Affiliated Children's Hospital

Dawei He

Chongqing Medical University Affiliated Children's Hospital

\section{Research Article}

Keywords: Piwil2-iCSCs, piRNA, NOP56, Proliferation, Migration, Invasion, Apoptosis

Posted Date: June 1st, 2021

DOI: https://doi.org/10.21203/rs.3.rs-439016/v1

License: (c) (1) This work is licensed under a Creative Commons Attribution 4.0 International License.

Read Full License 


\section{Abstract}

Objective Cancer stem cells (CSCs) play an important role in tumor development. Some studies have demonstrated that P-element-induced wimpy testis (Piwi)-interacting ribonucleic acids (piRNAs) participate in the progression of various cancers. However, the detailed function of piRNAs in CSCs requires further investigation. The aim of the present study was to investigate the effect of the uknown upregulated piRNA MW557525 and its predicted target gene nucleolar protein 56 (NOP56) inPiwi-like protein 2 (Piwil2)-induced CSCs (Piwil2-iCSCs).

Methods We screened differential piRNAs of Piwil2-iCSCs using high-throughput sequencing (HTS). Target genes were predicted by the miRanda algorithm and subjected to Gene Ontology (GO) analysis. One of the differential piRNAs, MW557525, and its target gene NOP56 were transfected and silenced in Piwil2-iCSCs, respectively. Quantitative real-time polymerase chain reaction (qRT-PCR) was conducted to detect expression levels of piRNA MW557525 and NOP56 in Piwil2-iCSCs after transfection. We measured protein levels of NOP56 in different groups via Western blot (WB), verified interactions using a dual luciferase reporter assay (LRA) and investigated the effect of piRNA MW557525 and NOP56 on Piwil2-iCSC proliferation using a Cell Counting Kit-8 (CCK-8). In addition, we evaluated cell migratory and invasive abilities via transwell assay and detected cell apoptotic ability via flow cytometry (FCM) assay. Protein levels of Cluster of Differentiation 24 (CD24), CD133, Krüppel-like factor 4 (KLF4) and sexdetermining region $\mathrm{Y}$-related high-mobility group (HMG) box 12 (SOX2) were measured to evaluate the change in Piwil2-iCSC pluripotency after transfection.

Results Via HTS, we screened out 204 differential piRNAs, and miRanda predicted 77 target genes. GO analysis showed that the biological processes (BPs) of these target genes were mainly involved in regulating the calcium concentration of cells and their molecular functions (MFs) were mainly involved in ATPase activity.The expression of piRNA MW557525 and NOP56 were significantly upregulated,and piRNA MW557525 was negatively associated with NOP56 in Piwil2-iCSCs. PiRNA MW557525 promoted proliferation, migration, invasion and pluripotency and inhibited apoptosis, while NOP56 suppressed proliferation, migration, invasion and pluripotency and induced apoptosis, in Piwil2-iCSCs.

Conclusion Taken together, these findings suggested that piRNA MW557525 promoted and maintained the vitality and pluripotency of Piwil2-iCSCs, while NOP56 inhibited these characteristics. Therefore, piRNA MW557525 might be a novel therapeutic target in Piwil2-iCSCs.

\section{Introduction}

P-element-induced wimpy testis (Piwi) protein of the Argonaute protein family is mainly expressed in germline stem cells of various organisms or embryonic/gonadal tumor tissues; it interacts with Piwiinteracting ribonucleic acid (piRNA), maintaining the stability of key conserved genes in germ cells [1]. Piwi-like proteins 2 and 4 (Piwil2 and Piwil4) and piRNA have been confirmed to be differentially expressed in a variety of tumor tissues, while the expression level and distribution of piRNA differ by 
tumor type [2-4]. Studies have found that piR-L-163 can directly bind and regulate phosphorylated ezrinradixin-moesin (ERM) and plays a key role in protein activation in lung cancer $[9,15]$. PiR-Hep1 is upregulated in liver cancer tumor tissues (46.6\%) and promotes tumor cell invasion, activity and migratory ability $[5,16,22]$. These differentially expressed piRNA also have potential for clinical use. PiR823 is significantly downregulated in gastric-cancer (GC) tissues, and its expression level has no correlation with the clinicopathological characteristics of GC. However, in cell and animal experiments, piR-823 inhibits the growth of GC cells, indicating that it is a potential therapeutic target in this cancer [23]. Yin et al. believe that piR-823 can promote the occurrence of colorectal cancer (CRC). Knockdown of this piRNA can induce cell G1 resistance and apoptosis to inhibit the viability of CRC cells. Therefore, piR823 could be a potential treatment target in CRC. Another study pointed out that the piR-932/Piwil2 complex accelerates the activity of breast cancer (BC) stem cells by promoting emulsion methylation, thereby promoting epithelial-mesenchymal transition (EMT). It is speculated that Piwil2 and piR-932 will become targets for blocking BC metastasis [24]. Therefore,clarifying the roles of piRNAs in various tumors will be helpful in tumor diagnosis and treatment.

Occurrence, drug resistance and metastasis of malignant tumors are all related to the presence of a small number of cancer stem cells (CSCs) in the tumor [6-7]. In current research, CSCs are generally obtained by isolating stem cell from solid tumor tissues, but there remain considerable difficulties in the extraction and culture of CSCs. In the early stage of this study, our research group established tumor-like stem cells by reprogramming human fibroblast (FB) genes with Piwi family protein Piwil2 [8]. We cultured these tumor-like stem cells for use in an in vitro tumor stem cell model; the differentially expressed piRNA regulated by Piwil2 was screened by high-throughput sequencing (HTS) technology, and its target gene was predicted. Analyzing the profile of differentially expressed piRNA and its predicted target genes and clarifying their effect on Piwil2-iCSCs would help us more comprehensively understand the mechanism of CSCs.

In this study, 204 differentially expressed piRNAs had 77 predicted target genes. Gene Ontology (GO) analysis of these target genes showed that their biological processes (BPs) mainly involved regulation of the calcium concentration in cells, and their molecular functions (MFs) mainly involved ATPase activity. We picked one of the differentially expressed piRNAs, piRNA MW557525, to conduct an in vitro cell assay. Results showed that this piRNA could promote cell proliferation, migration and invasion and inhibit apoptosis in Piwil2-iCSCs. As predicted by the miRanda algorithm

(http://cbio.mskcc.org/microrna_data/manual.html), the target gene of piRNA MW557525 was nucleolar protein 56 (NOP56), a yeast nucleolar protein that is part of a complex with the nucleolar proteins NOP58p and fibrillarin. NOP56p is required for assembly of the 60S ribosomal subunit and is involved in pre-ribosomal-RNA (pre-rRNA) processing. Silencing NOP56 promoted proliferation, migration and invasion and inhibited apoptosis in Piwil2-iCSCs. NOP56 was indirectly modulated by piRNA MW557525, and western blot (WB) results showed that piRNA MW557525 could increase the pluripotency of Piwil2iCSCs. 


\section{Materials And Methods \\ 2.1. Cells and cell culture}

The Piwil2-GFP-labeled lentiviruses were infected with the primary cultured pediatric foreskin FB, named Piwil2-iCSCs. In vitro cell chromosome karyotype analysis confirmed that Piwil2-iCSCs are tumor-like stem cells induced by Piwil2 [8], and they were stored in liquid nitrogen for a long period of time. For our experiments, we removed the Piwil2-iCSCs and FB cells from the $-80^{\circ} \mathrm{C}$ refrigerator, quickly rewarmed them in a $37^{\circ} \mathrm{C}$ water bath, transferred the cell suspension to a $10-\mathrm{mL}$ EP tube, added $2 \mathrm{~mL} \mathrm{F-12}$ medium and washed the mixture via centrifugation. Next, cells were cultured in F-12 medium containing $10 \%$ FBS in a $10-\mathrm{cm}$ petri dish and then in an incubator at $37^{\circ} \mathrm{C}$ with $5 \% \mathrm{CO}_{2}$. The medium was exchanged every 48 $\mathrm{h}$ and used for experiments when they grew to $>80 \%$ confluence.

\subsection{High-throughput sequencing and target gene prediction}

We sent both sets of cell samples, the Piwil2-iCSCs and the GFP-FBs, to Shanghai Huiyan Biotechnology Co., Ltd. (Shanghai, China) for second-generation sequencing. The Piwil2-iCSCs served as the experimental group, and the GFP-FBs served as the control group. Shanghai Huiyan constructed a complementary deoxyribonucleic acid (cDNA) library and performed HTS. Screening criteria for differential genes were $P<0.05$ and fold change $(F C) \geq 1.5$. We used the miRanda algorithm (http://www.microrna.org/microrna/getDownloads.do) to predict target genes of differential piRNAs. The target genes of piRNAs were analyzed by GO (http://www.geneontology.org) and KEGG(http://www.genome.jp/dbget-bin). The P-value ( $\mathrm{P}$ b 0.05) denotes the significance of $\mathrm{GO}$ terms enrichment in the DE genes and the Pathway correlated to the conditions. The lower the $p$-value, the more significant the GO Term and Pathway.

\subsection{PiRNA mimic/inhibitor and small interfering RNA (siRNA) transfection}

PiRNA MW557525 inhibitor, PiRNA MW557525 mimic and corresponding controls were synthesized by Shanghai GeneBio (Shanghai, China), from whom we also purchased NOP56 siRNA and negative control (NC). We plated Piwil2-iCSCs into six-well plates and transiently transfected them using Lipofectamine 2000 (Invitrogen, Carlsbad, CA, USA) per manufacturer's protocol. Sequences of these oligonucleotides are listed in Table 1.

\section{Table 1}

PiRNA MW557525 interference and NOP56 siRNA sequences 


\begin{tabular}{|c|c|}
\hline piRNA MW557525 interference & sequences(5'-3') \\
\hline piRNA MW557525 Mimics & 5'UGGAGGUGAUGAACUGUCUGAGCCUGACCUU-3' \\
\hline piRNA MW557525 Mimics NC & 5'UCACAACCUCCUAGAAAGAGUAGA-3' \\
\hline piRNA MW557525 Inhibitor & 5'AAGGUCAGGCUCAGACAGUUCAUCACCUCCA-3' \\
\hline piRNA MW557525 Inhibitor NC & 5'-UCUACUCUUUCUAGGAGGUUGUGA-3' \\
\hline NOP56 siRNA & 5'-UGGCAAACAAAUGCAGUAU-3' \\
\hline & 5'AUACUGCAUUUGUUUGCCA-3' \\
\hline
\end{tabular}

Total RNA from transfected Piwil2-iCSCs was extracted using TRIzol reagent (Invitrogen, Carlsbad, CA, USA). The isolated RNA was reverse transcribed using an miRcute miRNA FirstStrand Synthesis Kit (Tiangen, Beijing, China) for miRNA and Prime Script RT Master Mix (Takara, Kusatsu, Shiga, Japan) for mRNA according to the manufacturer's instructions. MiRNA quantity was measured using the miScript SYBR Green PCR Kit (Tiangen, Beijing, China), and mRNA quantity was measured using the QuantiNova ${ }^{\text {TM }}$ SYBR Green PCR Kit (Qiagen GmbH, Hilden, Germany). Reactions were performed on a CFX96 Real-Time PCR Detection System (Bio-Rad, USA). The PCR conditions were $30 \mathrm{~s}$ at $95^{\circ} \mathrm{C}$, followed by 40 cycles at $95^{\circ} \mathrm{C}$ for $5 \mathrm{~s}$ and $60^{\circ} \mathrm{C}$ for $45 \mathrm{~s}$. The relative expression levels of piRNA MW557525 and NOP56 mRNA were calculated by the comparative cycle threshold $(\mathrm{Ct})$ method using the expression of U6 small nuclear RNA as the reference for miRNA and GAPDH as the reference for mRNA. The $2^{-} \Delta \Delta \mathrm{ct}$ method was used for analysis. The sequence-specific primers used for piRNA MW557525, U6, NOP56 and GAPDH are shown in Table 2.

Table 2

Sequence-specific primers.

\begin{tabular}{|ll|}
\hline primers & sequences(5'-3') \\
\hline piRNA MW557525 & GGAGGTGATGAACTGTCTGAGCCTGACCTT \\
\hline NOP56 & Fwd:TGAAGATCATCAACGACAATGC \\
& Rev:TTCAGATAAAGACACCACACGA \\
\hline The internal reference primer U6 and GAPDH are brought from Sangon Biotech (Shanghai) Co., Ltd. \\
\hline 2.5. Western blotting
\end{tabular}

Total proteins from transfected Piwil2-iCSCs were isolated using RIPA buffer (Beyotime, China) supplemented with phenylmethanesulfonyl fluoride (PMSF), and concentrations were checked via 
bicinchoninic acid (BCA) assay. We separated the proteins using sodium dodecyl sulfate polyacrylamide gel electrophoresis (SDS-PAGE) and then transferred the electrophoretic bands onto polyvinylidene fluoride (PVDF) membranes (MilliporeSigma, Burlington, MA, USA). Next, the membranes were blocked with $5 \%$ bovine serum albumin (BSA; ZSGB-BIO Technology Co., Ltd., Beijing, China)/tris-buffered saline + Polysorbate 20 (TBST) for $1 \mathrm{~h}$ at room temperature (RT). We used an NOP56 mouse antibody (1:1000; Abcam, Cambridge, UK), Cluster of Differentiation 24 (CD24) rabbit antibody (1:500; Santa, USA), CD133 rabbit antibody (1:1000, Abcam), Krüppel-like factor 4 (KLF4) rabbit antibody (1:1000; Chengdu Zen Bioscience Co., Ltd., Chengdu, China), sex-determining region $Y$-related high-mobility group (HMG) box 12 (SOX2) mouse antibody (1:1000; Abcam), B-cell lymphoma 2 (BCl-2)-like protein 4 (BAX) mouse antibody (1:1000), $B c l-2$ mouse antibody (1:1000) and GAPDH rabbit antibody (1:1000; all three from Chengdu Zen) as primary antibodies. After incubation with primary antibodies overnight at $4^{\circ} \mathrm{C}$, we washed the membranes with TBST three times for at least 10 min per wash at RT. The membranes were then incubated with secondary antibody at RT for $1 \mathrm{~h}$, followed by three washes with TBST for 10 min each. We analyzed the membranes using a ChemiDoc MP Imaging System (Bio-Rad Laboratories, Hercules, CA, USA) after treating them with an Electrochemiluminescence (ECL) Kit (Vazyme Biotech Co., Ltd., Nanjing, China).

\subsection{Cell Counting Kit-8 (CCK-8) assay}

Cell proliferation assays were performed using Cell Counting kit-8 assays (MCE, China) following the manufacturer's protocol. Approximately $3 \times 10^{3}$ Piwil2-iCSCs were plated in 96-well plates and transfected with mimics, inhibitor, NOP56 siRNA, or NC oligonucleotides. At the indicated time points (days $0,1,2$ and 3 ), we removed the culture medium and added $100 \mu \mathrm{L}$ CCK-8 medium to each well. Cells were incubated for an additional $2 \mathrm{~h}$, and optical density (OD) was measured at an absorbance wavelength of $450 \mathrm{~nm}$ on a microplate reader (Bio-Rad, USA).

\subsection{Flow cytometry assay}

An FITC Annexin V Apoptosis Detection Kit I (BD Pharmingen ${ }^{\text {TM }}$, USA) was used to check cell apoptosis following the manufacturer's instructions. $5 \times 10^{5}$ Piwil2-iCSC cells transfected with mimics, inhibitor, NOP56 siRNA, or negative control were trypsinized and washed twice with phosphate-buffered saline (PBS). The cells were resuspended using the binding buffer, and then, $5 \mu \mathrm{L}$ Annexin V-fluorescein isothiocyanate (FITC) and $5 \mu \mathrm{L}$ propidium iodide (PI) were added to the buffer and the mixture incubated for $15 \mathrm{~min}$ in the dark. We analyzed the stained cells on a flow cytometer (BD Biosciences, USA), and the apoptosis rate was analyzed using FlowJo software (BD Biosciences).

\subsection{Transwell assay}

Transwell chambers precoated with Matrigel (1:8, BD, USA) were employed to check the capacity of cell invasion. Piwil2-iCSCs $(1 \times 105$ cells suspended in $200 \mu \mathrm{L}$ of culture medium without FBS $)$ transfected with mimics, inhibitor, NOP56 siRNA, or negative control were seeded into the upper chamber. Meanwhile, $600 \mathrm{uL}$ of medium containing 10\% FBS was added to the lower chamber. According to their varying 
migratory abilities, cells were returned to the incubator and permitted to culture for $24 \mathrm{~h}$. Next, we removed the transwell chambers and washed them three times each with $1 \times$ PBS. Then, the cells were fixed with $4 \%$ paraformaldehyde for $15 \mathrm{~min}$ and stained with $0.5 \%$ crystal violet solution for $30 \mathrm{~min}$. Cells on the upper surfaces of the chambers were carefully wiped off with water and cotton swabs. Images were taken in five randomly selected fifields using a microscope.The transwell migration assay was conducted identically to the transwell invasion assay, except that the upper chamber was not coated with Matrigel. These experiments were independently carried out three times each.

\subsection{Dual luciferase reporter assay (LRA)}

Bioinformatics prediction algorithms were used to show that the 3'-UTR of NOP56 contained potential binding sites for piRNA MW557525. We inserted the wild-type (WT) sequence of NOP56 3'-untranslated region (UTR) harboring the binding sites of piRNA MW557525 into the pGL3 vector (Shanghai GeneBio) to construct the luciferase reporter vector NOP56 3'-UTR-WT. Similarly, we established NOP56 3'-UTRmutated (MUT) reporter vector by mutating the potential target sites of the piRNA. Piwil2-iCSCs were cotransfected with the recombinant-vector piRNA mimic and NC plasmid using Lipofectamine 2000 . We measured Firefly and Renilla luciferase activity $48 \mathrm{~h}$ after cotransfection using a Dual LRA Kit (Promega Corp., Fitchburg, WI, USA) per manufacturer's instructions and calculated the values based on the activity of the Renilla/Firefly luciferase genes.

\subsection{Statistical analysis}

We performed calculations of experimental data using Graph Pad Prism 8.0.1(GraphPad, San Diego, CA, USA) and presented the data as means \pm standard deviations (SDs). Two independent groups were compared by using Student's $t$ test. One-way analysis of variance (ANOVA) was followed by a Bonferroni post hoc test for multiple groups or an unpaired, two-tailed Student's t-test for two groups. Each experiment was independently repeated at least three times.

\section{Results}

\subsection{PiRNA expression profile and target gene prediction}

We screened 204 differentially expressed piRNAs from Piwil2-iCSCs and compared them with the piRNA Database (http://www.regulatoryrna.org/database/piRNA/). We found that 159 of them had been discovered and reported (45 in human genes, 114 in non-human genes), and the remaining 45 were new, unreported piRNAs. Of all 204 differentially expressed piRNAs, 121 (22 of which were unknown) were significantly upregulated, and 83 (28 of which were unknown) were significantly downregulated, in Piwil2iCSCs (Fig. 1A). We used ND to name the unknow downregulated piRNAs, and NU was uesd to name the unknown upregulated piRNA.piRNA MW557525 was one of the unkown upregulated piRNAs. Then we used the miRanda algorithm to predict target genes of the 204 differentially expressed genes. From a total of 74 piRNAs, a total of 77 target genes were predicted. We found that NOP56 was the predicted taget gene of piRNA MW557525. GO analysis of the latter $(P \leq 0.05)$ via DAVID found that their BPs 
might be mainly involved in regulating cytosolic calcium ion concentration, and their MFs might be mainly involved in ATPase activity (Fig. 1B). KEGG results showed that the adenosine triphosphate (ATP)-binding cassette (ABC) transporter pathway was the most likely pathway of these genes' BPs and MFs (Fig. 2C). We used the Search Tool for the Retrieval of Interacting Genes/Proteins (STRING; www.string-db.org) to carry out protein interaction network analysis (interaction score 0.15 ). Among the 77 predicted target genes, myosin IXa (MYO9A) and protein tyrosine phosphatase receptor type delta (PTPRD) each had seven interacting proteins, representing the strongest interaction points. The highest combined-score pair was RPS8 and RPS29 (0.999); the second-highest was RPS8 and NOP56 (0.994; Fig. 1D).

A:Heatmap of differentially expressed piRNA in Piwil2-iCSCs. The difference between duplicate groups may be due to measurement errors.B-C: GO functional classification and KEGG analysis of predicted target genes of differentially expressed piRNAs in Piwil2-iCSCs. Regulation of cytosolic calcium ion concentration, ATPase activity, and ABC transporters pathway were the most possible processes involved in these target genes. The results of $G O$ analysis showed that the $p$ value was greater than 0.05 , so the results were only for reference.

D:Protein interaction analysis of predicted target genes of differentially expressed piRNAs in Piwil2iCSCs. The higher combined score meaned higher credibility and proteins with more interaction points were more important in PPI.The highest combined score pairs were RPS8 and RPS29. PTPRD and MY09A had the most interaction points.

\subsection{Expression of piRNA MW557525/NOP56 and their successful transfection in Piwil2-iCSCs}

We measured the expression of piRNA MW557525 and NOP56 in Piwil2-iCSCs versus FBs. The results showed that the levels of piRNA MW557525 and NOP56 were significantly upregulated (Fig. 2A-B). Next, we detected the expression of piRNA MW557525 and NOP56 after transfection with MW557525 mimic, inhibitor, NOP56 siRNA and corresponding NC plasmid to confirm transfection efficiency. PiRNA MW557525 mimic transfection increased piRNA MW557525 expression, while PiRNA MW557525 inhibitor transfection decreased piRNA MW557525 expression (Fig. 2C). In addition, siRNA NOP56 transfection decreased mRNA and protein levels of NOP56 (Fig. 2D-E).

\subsection{PiRNA MW557525 was negatively associated with NOP56 in Piwil2-iCSCs}

To confirm whether piRNA MW557525 was associated with NOP56, we examined its effect on NOP56 expression by detecting NOP56 mRNA and piRNA MW557525 levels $48 \mathrm{~h}$ after transfection. Upregulation of piRNA MW557525 decreased mRNA and protein levels of NOP56, while downregulation thereof increased these levels (Fig. 3A-B). Furthermore, siRNA transfection increased the mRNA levels of piRNA MW557525 (Fig. 3C). Therefore, we speculated that piRNA MW557525 might modulate NOP56 
expression. Bioinformatics prediction algorithms showed that the 3'-UTR of NOP56 contained potential binding sites for piRNA MW557525 (Fig. 3D). Then, we performed a dual LRA to confirm this prediction, and the data indicated that piRNA MW557525 did not significantly diminish the luciferase activity of NOP56 3'-UTR-WT versus NOP56 3'-UTR-MUT in Piwil2-iCSCs (Fig. 3E). Taken together, these results showed that piRNA MW557525 was negatively associated with NOP56 in Piwil2-iCSCs.

\subsection{PiRNA MW557525 promoted proliferation, migration and invasion and inhibited apoptosis in Piwil2-iCSCs}

To investigate cellular biological changes of piRNA MW557525 in Piwil2-iCSCs, we used CCK-8, transwell, FCM and WB assays to detect cell proliferation, migration, invasion and apoptosis, respectively. Results showed that the proliferative ability of Piwil2-iCSCs in the mimic transfection group was remarkably higher, and their apoptotic ability lower, than in the NC group. Downregulation of piRNA MW557525 suppressed cell proliferation and induced apoptosis (Fig. 4A-C). Upregulation of piRNA MW557525 via piRNA MW557525 mimic transfection significantly promoted Piwil2-iCSC cell migration (Fig. 4D) and invasion (Fig. 4E), while downregulation of piRNA MW557525 yielded the opposite results. Taken together, these results strongly supported the hypothesis that piRNA MW557525 might help promote the occurrence and progression of Piwil2-iCSCs.

\subsection{NOP56 inhibited proliferation, migration and invasion and promoted apoptosis in Piwil2-iCSCs}

We then performed cell functional experiments to detect the effect of NOP56 on Piwil2-iCSC proliferation, migration, invasion and apoptosis. Results showed that the NOP56 silencing group had remarkably higher proliferative ability than the NC group, and that silencing NOP56 suppressed Piwil2-iCSC apoptosis (Fig. 5A-C). Knockdown of NOP56 via NOP56 siRNA promoted Piwil2-iCSC migration (Fig. 5D) and invasion (Fig. 5E) compared with the NC group. These results suggested that NOP56 inhibited the occurrence and progression of Piwil2-iCSCs.

\subsection{PiRNA MW557525 promoted the pluripotency of Piwil2-iCSCs, while NOP56 suppressed the pluripotency of Piwil2-iCSCs}

Piwil2-iCSCs act as tumor-like stem cells, the most important characteristic is pluripotency. We performed WB to detect changes in the pluripotency of piRNA MW557525 and NOP56 in Piwil2-iCSCs. As CD24, CD133, KLF4 and SOX2 are markers of stem cell pluripotency, we detected their expression levels. Compared with the NC group, overexpression of piRNA MW557525 increased the expression of CD24, CD133, KLF4, SOX2, while downregulation of piRNA MW557525 yielded the opposite results (Fig. 6A). In addition, NOP56 knockdown also upregulated the expression of CD24, CD133, KLF4 and SOX2 compared 
with NC (Fig. 6B). Taken together, these results showed that PiRNA MW557525 promoted the pluripotency of Piwil2-iCSCs, while NOP56 suppressed it.

\section{Discussion}

CSCs have the characteristics of carcinogenicity, multiple differentiation and self-renewal. Some scholars believe that tumor recurrence, metastasis and resistance to radiotherapy and chemotherapy were due to the existence of tumor stem cells [10]. The first solid tumor to prove the existence of CSCs was BC; Al-Hajj et al. proved that $C D 44^{+} / C D 24^{-}$/(low)-lineage cells isolated from $\mathrm{BC}$ tissue can form tumors in immunodeficient mice [25]. Studies have shown that in CRC, CSCs expressing $C D 133^{+}$and $C D 44^{+} / C D 166^{+}$are more resistant to treatment [26]; $C D 133$ is also a surface marker of pancreatic-cancer (PC) stem cells $[29,30]$. CSCs rich in $C D 133^{+}$and $C D 44^{+} / \mathrm{CMet}^{+}$in $\mathrm{PC}$ are also more prone to drug resistance [31]. This research on CSCs is expected to be applied to clinical treatment. Hedgehog $(H h)$, Wingless/Integrated (Wnt), Notch and nuclear factor K-light-chain-enhancer of activated B cells (NF-KB) pathway inhibitors can inhibit the renewal of PC tumor stem cells; the pluripotency of stem cell factors such as Pituitary-Octamer-Unc-86 (POU) class 5 homeobox 4 (OCT4), SOX2, cellular myelocytomatosis oncogene $(c-M y c)$ and Nanog, and tumor growth in vivo and in vitro [32]. CD133 $/ C D 44^{+}$liver cancer stem cells show stem cell characteristics such as extensive proliferation, self-renewal and the ability to differentiate into cancer cells. Therefore, some researchers believe blocking CD44 signal transduction with anti-CD44 antibodies could be a potential strategy to eradicate hepatic CSCs and cure patients [33]. Exploring the origin and markers of CSCs involved in the occurrence and growth will help us better judge the prognoses of patients with tumors and more accurately target therapies. In the early stage of this study, our group used Piwil2 to reprogram human FBs in order to establish Piwil2-iCSCs that stably expressed Piwil2-GFP. The cell biological-behavior test and tumor stem cell marker test confirmed that the reprogrammed cells were tumor-like stem cells [8], which could be used in an ideal in vitro CSCs model.

A piRNA is a small non-coding RNA (sncRNA) 23-31 nt long; it is characterized by common monophosphate uracil at the 5 '-end and methylation modification at the 3 '-end [11]. After piRNA is combined with Piwi proteins (including Piwil2 and Piwil4), it inhibits the function of transposon via the ping-pong effect and stabilizes genes. In addition, piRNA can participate in gene regulation via DNA methylation and histone modification [12-14]. Many studies have proven that abnormally expressed piRNA is related to lung, liver, stomach, colorectal, bladder and breast cancers.

In the in vitro CSCs model constructed by reprogramming FBs with Piwil2, we speculated that the expression and functional changes of related piRNAs caused by overexpression of the Piwil2 gene might play important roles in the process of tumorigenesis, and the differentially expressed piRNA might affect tumor tissues. Therefore, the differentially expressed piRNAs could be of great value for exploring the mechanism underlying the occurrence and promotion of CSCs.

In this study, we screened 204 differentially expressed piRNAs of Piwil2-iCSC using HTS technology, and a total of 77 target genes were predicted by the miRanda algorithm. GO analysis of the selected target 
genes with differential expression of piRNAs revealed that the main BP regulated cell calcium ion concentration and the main MF participated in ATPase activity. After we used STRING to analyze the protein interaction network, the results showed the highest combined score of interaction between RPS8 and RPS29 (0.999) and the second-highest between RPS8 and NOP56 (0.994). MYO9A and PTPRD each had seven interacting proteins, representing the strongest interaction points. Based on these bioinformatics data, we speculated that the role of piRNA in CSCs might be related to energy metabolism. Many studies have shown that tumors and their stem cells have abnormalities in energy metabolism. As they have significantly fewer mitochondriaes than normal cells, tumors and CSCs mainly produce energy via glycolysis $[27,28]$. It is speculated that piRNA regulates the energy metabolism of CSCs by targeting its target genes or by some other mechanism.

Next, we chose piRNA MW557525 to further study the function of piRNAs in CSCs. Via WB, qRT-PCR, CCK8 , Transwell and FCM assays, we found that piRNA MW557525 could promote proliferation, migration and invasion and inhibit apoptosis in Piwil2-iCSCs, which suggested that it could trigger CSCs occurrence and progression. By detecting the expression of CD24,CD133, KLF4 and SOX2 via WB, we found that piRNA MW557525 could upregulate these markers of stem cell pluripotency, meaning that it could increase Piwil2-iCSCs pluripotency and decrease CSCs differentiation. Therefore, we think that piRNA MW557525 could be an important factor in the occurrence and maintenance of CSCs. Interestingly, we detected high expression of NOP56 in Piwil2-iCSC. Regarding this phenomenon, we believe that increasing the amount of Piwil2 in cells not only changes the expression of MW557525, but also regulates other genes that can target NOP56, thereby increasing the expression of NOP56 at the overall level.

Via the miRanda algorithm, we predicted NOP56 as the target gene of piRNA MW557525. NOP56 participates in the modification of rRNA precursor ribose methylation and is a component of the smallmolecule nucleolar ribonucleoprotein (snoRNP) complex [19]. Studies have found that its abnormality is related to the Myc gene mutation in Burkitt lymphoma [20]. Some scholars have shown that NOP56 could be a promising potential therapeutic target in CRC [21]. In our study, silencing NOP56 could promote proliferation, migration and invasion and inhibit the apoptosis of Piwil2-iCSCs. We found that upregulation of MW557525 decreased the expression of NOP56 and vice versa, and a dual LRA confirmed that NOP56 was negatively related by piRNA MW557525 and indirectly modulated by MW557525.

Many studies have shown that Piwi and piRNA regulate embryogenesis and somatic-cell development in many organisms. The ping-pong effect of piRNA and Piwi protein can consume transposable factors, thereby ensuring the stability of key genes. The piRNA pathway has been proven to influence cell stemness in primitive animals with high regenerative ability, as well as in germ lines and somatic stem cells of other animals [34,35]. In planarians and ascidians, Piwi is an essential gene for maintaining function in pluripotent stem cells (PSCs) [36]. In Drosophila, Piwi affects somatic-cell function through epigenetic and post-transcriptional regulation [37, 38]. Piwi/piRNA complex has also been shown to be related to brain development, memory formation and translation of neuronal damage [39]. Meanwhile, 
some scholars believe that the Piwi protein family can enhance the pluripotency of mammalian cells, but it is dispensable for mouse somatic development and for reprogramming FBs into PSCs [40]. The results of this study showed that upregulating piRNA MW557525 alone could enhance the stemness of cells. We speculate that piRNA maintains cell stemness through Piwi/piRNA complex in vitro, and that it has other ways of doing so such as direct or indirect regulation of targeted genes.

In addition to targeting related genes, piRNA has many other means of regulating the genes of tumor/cancer stem cells. Many studies have reported that piRNA is involved in tumorigenesis, most commonly by regulating the function of transposons by combining with Piwi protein to affect tumorigenesis [17]. In addition, piRNA and Piwi/piRNA complex can also regulate tumor growth by targeting certain protein-coding genes, such as recruiting DNA methyltransferase (DNMT) to regulate DNA methylation. [18]. Therefore, we speculate that piRNA MW557525 indirectly affects NOP56 by other means or through other elements, rather than simply targeting it directly.

This research innovatively sequenced cancer stem cells constructed artificially to obtain unknown differentially expressed piRNA.MW557525 is one of the unknown differentially expressed piRNA and its target gene is predicted to be NOP56.We conduct cell experiments to prove the function of unknown piRNA and its relationship with the predicted target gene NOP56.In conclusion, our research demonstrated that piRNA MW557525 could promote and maintain the vitality and pluripotency of Piwil2iCSCs while NOP56 inhibited the vitality and the stemness of Piwil2-iCSCs.PiRNA MW557525 was negatively associated with NOP56 in Piwil2-iCSCs.Silencing the target gene NOP56 could also promote and maintain the vitality and pluripotency of Piwil2-iCSCs. This finding provides a novel focus, piRNA, in the exploration of CSCs' mechanism. We next plan to research the process by which piRNA MW557525 targets NOP56, as well as MW557525's effect on normal or cancer cells.

\section{Declarations}

\section{Author Declarations}

Ethics approval and consent to participate

All procedures performed in studies involving human participants were in accordance with the ethical standards of the institutional and/or national research committee and with the 1964 Helsinki declaration and its later amendments or comparable ethical standards. This article does not contain any studies with animals performed by any of the authors. The study protocol was approved by the Medical Ethics Committees of the participating institutions.

Consent for publication

Written informed consent for publication was obtained from all participants.

Availability of data and materials 
The datasets used or analyzed during the current study are available from the corresponding author on reasonable request.

Competing interests

The authors declare no competing interests.

Funding

Funding support is from Chongqing Science and Technology Commission Project (7000025).

Authors' contributions

ZAHOXIA ZHANG and ZHANG WANG performed the experiments, analyzed the data, and cowrote the manuscript. LIMING JIN, XIAOJUN TAN, and ZHAOYING WANG participated in the completion of the experiment. LIANJU SHEN, CHUNLAN LONG educate experimental techniques. GUANGHUI WEI, DAWEI HE supervised the work.

Acknowledgements

We gratefully acknowledge the assistance of Prof. GUANGHUI WEI and Prof. DAWEI HE in education and providing experiment platform.

\section{Compliance with Ethical Standards}

Disclosure of potential conflicts of interest

We have no potential conflict of interest.

Research involving Human Participants and/or Animals

Not applicable

Informed consent

Not applicable

\section{References}

1. TÓTH K F, PEZIC D (2016) STUWE E, WEBSTER A. The piRNA pathway guards the germline genome against transposable elements[J]. Adv Exp Med Biol 886:51-77

2. LIU J, ZHANG S (2018) CHENG B. Epigenetic roles of PIWI-interacting RNAs (piRNAs) in cancer metastasis (review)[J]. Oncol Rep 40:2423-2434

3. HIRAKATA S, SIOMI M C (2016) piRNA biogenesis in the germline: From transcription of piRNA genomic sources to piRNA maturation[J]. Biochim Biophys Acta 1859:82-92 
4. ZENG G, ZHANG D, LIU X, KANG Q, FU Y, TANG B et al (2017) Co-expression of Piwil2/Piwil4 in nucleus indicates poor prognosis of hepatocellular carcinoma[J]. Oncotarget 8:4607-4617

5. Kevin W, Ng C, Anderson EA, Marshall et al (2016) Piwi interacting RNAs in cancer: emerging functions and clinical utility. Mol Cancer 15:5

6. EUN K, HAM S W KIMH (2017) Cancer stem cell heterogeneity: origin and new perspectives on CSC targeting[J]. BMB Rep 50:117-125

7. PEIRIS-PAGÈS M, MARTINEZ-OUTSCHOORN U E, PESTELL R G, SOTGIA F (2016) LISANTI M P. Cancer stem cell metabolism[J/OL]. Breast Cancer Res 18:55. doi:10.1186/s13058-016-0712-6

8. ZHANG D, WU X, LIU X, CAI C, ZENG G, ROHOZINSKI J et al (2017) Piwil2-transfected human fibroblasts are cancer stem cell-like and genetically unstable[J]. Oncotarget 8:12259-12271

9. Mei Y, Wang Y, Kumari $P$ et al (2015) A piRNA-like small RNA interacts with and modulates p-ERM proteins in human somatic cells. Nat Commun 6:7316

10. SUN J, LI G, LIU Y, MA M, SONG K, LI H et al (2020) Targeting histone deacetylase SIRT1 selectively eradicates EGFR TKI-resistant cancer stem cells via regulation of mitochondrial oxidative phosphorylation in lung adenocarcinoma[J]. Neoplasia 22:33-46

11. ROJAS-RÍOS P SIMONELIGM. piRNAs and PIWI proteins: regulators of gene expression in development and stem cells[J/OL]. Development, 2018, 145. pii: dev161786. doi:

10.1242/dev.161786

12. OZATA DM, GAINETDINOV I, ZOCH A, O'CARROLL D (2019) ZAMORE P D. PIWl-interacting RNAs: small RNAs with big functions[J]. Nat Rev Genet 20:89-108

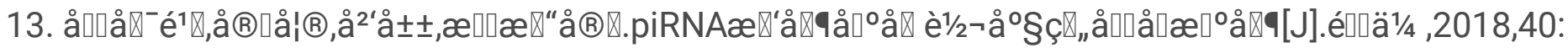
$445-450$

14. YIN J, JIANG X Y, QI W, JI C G, XIE X L, ZHANG D X et al (2017) piR-823 contributes to colorectal tumorigenesis by enhancing the transcriptional activity of HSF1[J]. Cancer Sci 108:1746-1756

15. Neisch AL, Fehon RG (2011) Radixin Ezrin.Moesin, key regulators of membrane-cortex interactions and signaling. Curr Opin Cell Biol 23:377-382

16. Law PT, Qin H, Ching AK et al (2013) Deep sequencing of small RNA transcriptome reveals novel noncoding RNAs in hepatocellular carcinoma. J Hepatol 58(6):1165-1173

17. LIU Y, DOU M, SONG X, DONG Y, LIU S (2019) LIU H, et al. The emerging role of the piRNA/piwi complex in cancer[J/OL]. Mol Cancer 18:123. doi:10.1186/s12943-019-1052-9

18. FU A, JACOBS D I, HOFFMAN A E, ZHENG T, ZHU Y (2015) PIWl-interacting RNA 021285 is involved in breast tumorigenesis possibly by remodeling the cancer epigenome[J]. Carcinogenesis 36:10941102

19. LYKKE-ANDERSEN S, ARDAL B K, HOLLENSEN A K, DAMGAARD C K, JENSEN TH, Box (2018) C/D snoRNP Autoregulation by a cis-Acting snoRNA in the NOP56 Pre-Mrna[J/OL]. Mol Cell 72:99111.e5. doi:10.1016/j.molcel.2018.08.017 
20. COWLING V H, TURNER S A, COLE MD (2014) Burkitt's lymphoma-associated c-Myc mutations converge on a dramatically altered target gene response and implicate Nol5a/Nop56 in oncogenesis[J]. Oncogene 33:3519-3527

21. Grade M, Hummon AB, Camps J, Emons G, Spitzner M, Gaedcke J, Hoermann P, Ebner R, Becker H, Difilippantonio MJ, Ghadimi BM, Beissbarth T, Caplen NJ, Ried T (2011) A genomic strategy for the functional validation of colorectal cancer genes identifies potential therapeutic targets. Int $\mathrm{J}$ Cancer. Mar 1;128(5):1069-79

22. Mei Y, Clark D, Mao L (2013) Novel dimensions of piRNAs in cancer. Cancer Lett 336(1):46-52

23. Cheng J, Deng H, Xiao B et al (2012) piR-823, a novel noncoding small RNA, demonstrates in vitro and in vivo tumor suppressive activity in human gastric cancer cells. Cancer Lett 315:12-17

24. Zhang H, Ren $\mathrm{Y}$, Xu H,et al (2013) The expression of stem cell protein Piwil2 and piR-932 in breast cancer. Surg Oncol 22:217-223

25. Al-Hajj M, Wicha MS, Benito-Hernandez A et al (2003) Prospective identification of tumorigenic breast cancer cells. Proc Natl Acad Sci USA 100:3983-3988

26. Todaro M, Alea MP, Di Stefano AB et al (2007) Colon cancer stem cells dictate tumor growth and resist cell death by production of interleukin-4. Cell Stem Cell 1:389-402

27. PALORINI R, VOTTA G, BALESTRIERI C, MONESTIROLI A, OLIVIERI S, VENTO R et al (2014) Energy metabolism characterization of a novel cancer stem cell-like line 3AB-OS[J]. J Cell Biochem 115:368-379

28. AMINZADEH S, VIDALI S, SPERL W (2015) KOFLER B, FEICHTINGER R G. Energy metabolism in neuroblastoma and Wilms tumor[J]. Transl Pediatr 4:20-32

29. Li C, Heidt DG, Dalerba P et al (2007) Identification of pancreatic cancer stem cells. Cancer Res 67:1030-1037

30. Simeone DM (2008) Pancreatic cancer stem cells: implications for the treatment of pancreatic cancer. Clin Cancer Res 14:5646-5648

31. Li C, Wu JJ, Hynes M et al (2011) c-Met is a marker of pancreatic cancer stem cells and therapeutic target. Gastroenterology 141:2218-2227

32. Ricardo Leão 1, Célia Domingos, A, Figueiredo,et al. Cancer Stem Cells in Prostate Cancer: Implications for Targeted Therapy. Urol Int. 2017;99(2):125-136

33. Zhu Z, Hao X, Yan M,et al (2010) Cancer stem/progenitor cells are highly enriched in CD133 + CD44 + population in hepatocellular carcinoma. Int J Cancer 126:2067-2078

34. Girard A, Sachidanandam R, Hannon GJ et al. A germline-specific class of small RNAs binds mammalian Piwi proteins. Nature. 2006 Jul 13;442(7099):199-202

35. Grivna ST, Beyret E, Wang Z, Lin H. A novel class of small RNAs in mouse spermatogenic cells. Genes Dev. 2006 Jul 1;20(13):1709-14

36. Juliano C, Wang J, Lin H (2011) Uniting germline and stem cells: the function of Piwi proteins and the piRNA pathway in diverse organisms. Annu Rev Genet 45:447-469 
37. Yin H, Lin H (2007) An epigenetic activation role of Piwi and a Piwi-associated piRNA in Drosophila melanogaster. Nature 450:304-308

38. Brower-Toland B, Findley SD, Jiang L, Liu L, Yin H et al (2007) Drosophila PIWI associates with chromatin and interacts directly with HP1a. Genes Dev 21:2300-2311

39. Kim KW. PIWI Proteins and piRNAs in the Nervous System. Mol Cells. 2019 Dec 31;42(12):828-835

40. Cheng EC, Kang D, Wang Z, Lin H (2014 Sep) PIWI proteins are dispensable for mouse somatic development and reprogramming of fibroblasts into pluripotent stem cells. PLoS One 19(9):e97821 9(

\section{Figures}




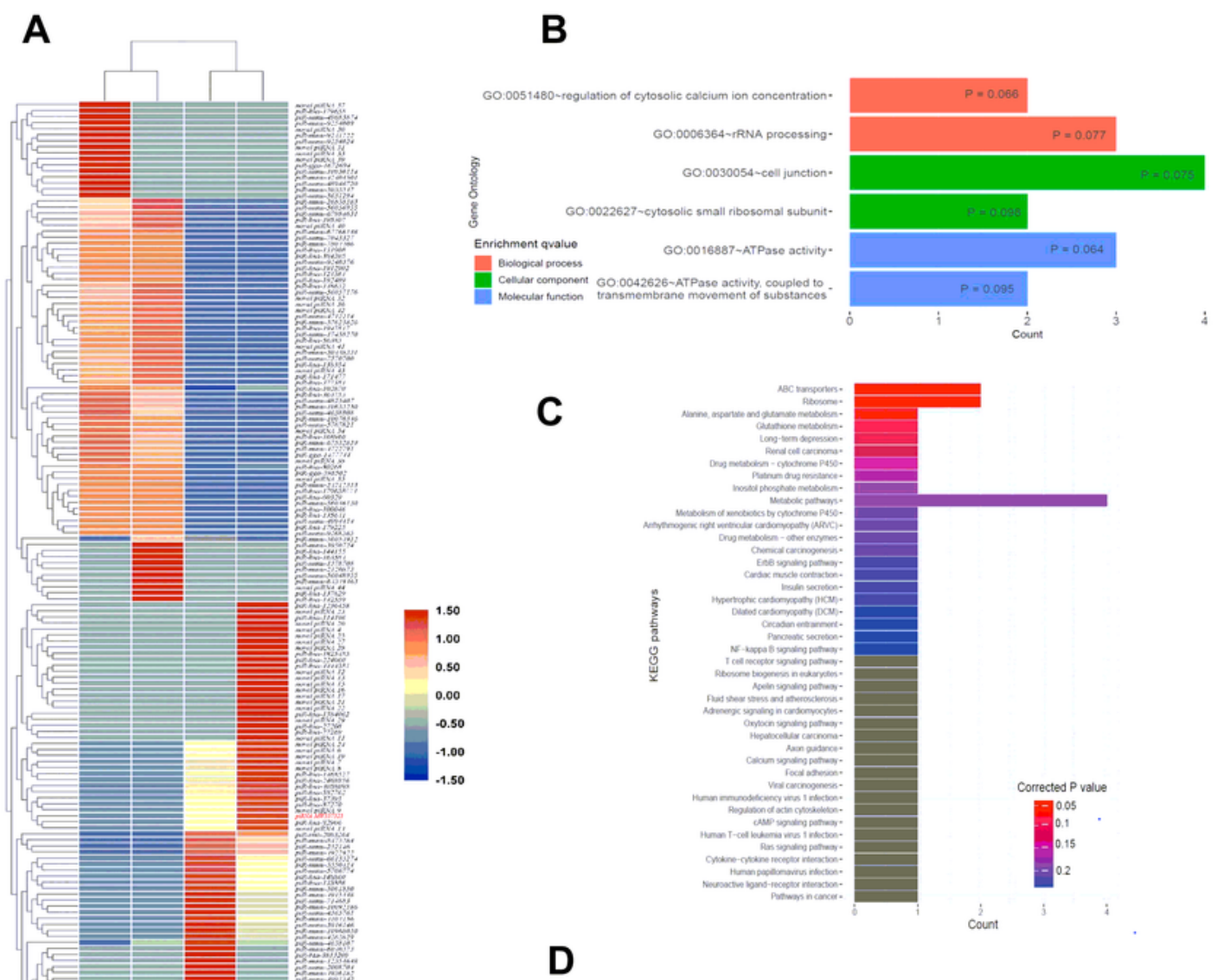


results of GO analysis showed that the $p$ value was greater than 0.05 , so the results were only for reference. D:Protein interaction analysis of predicted target genes of differentially expressed piRNAs in Piwil2-iCSCs. The higher combined score meaned higher credibility and proteins with more interaction points were more important in PPI.The highest combined score pairs were RPS8 and RPS29. PTPRD and MYO9A had the most interaction points.
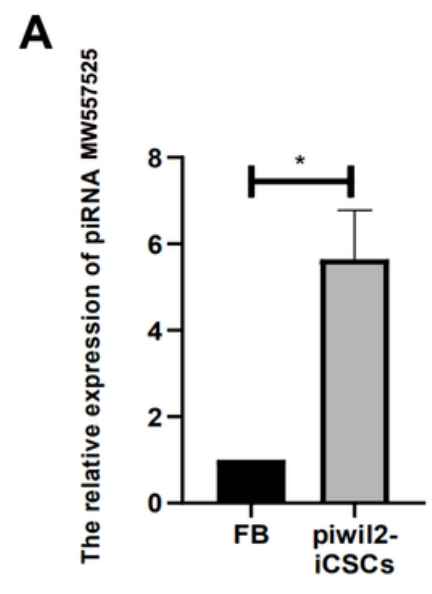

B
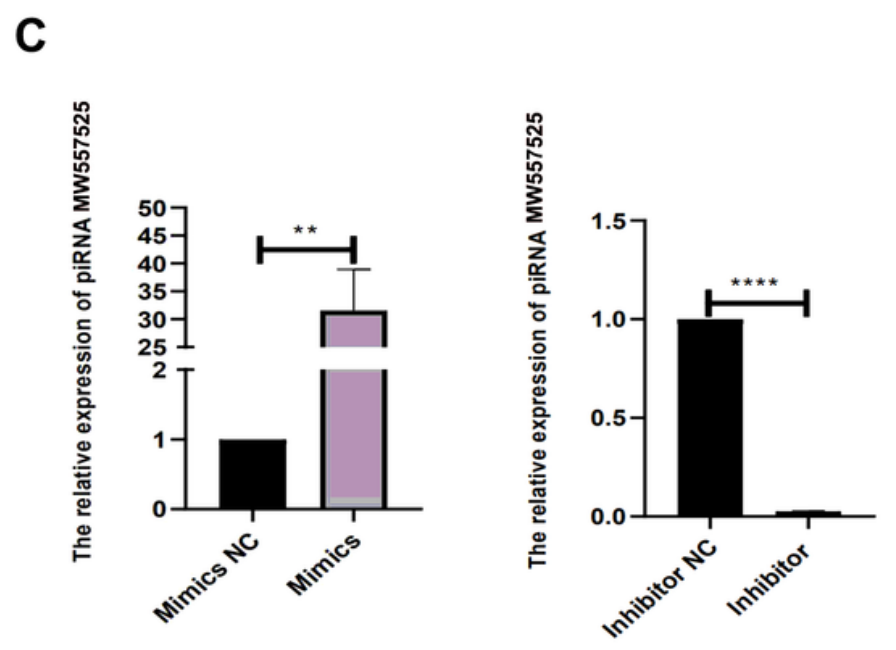

D

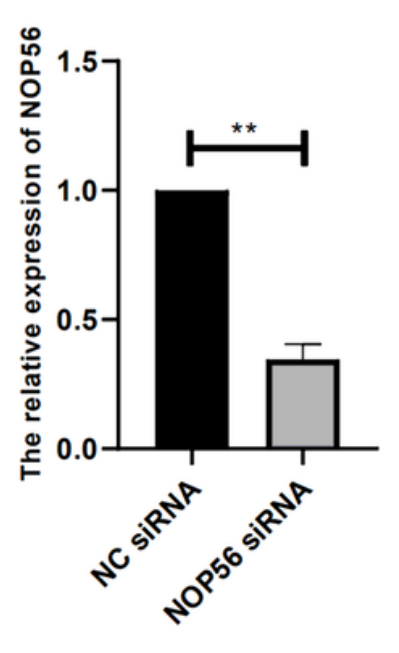

$E$
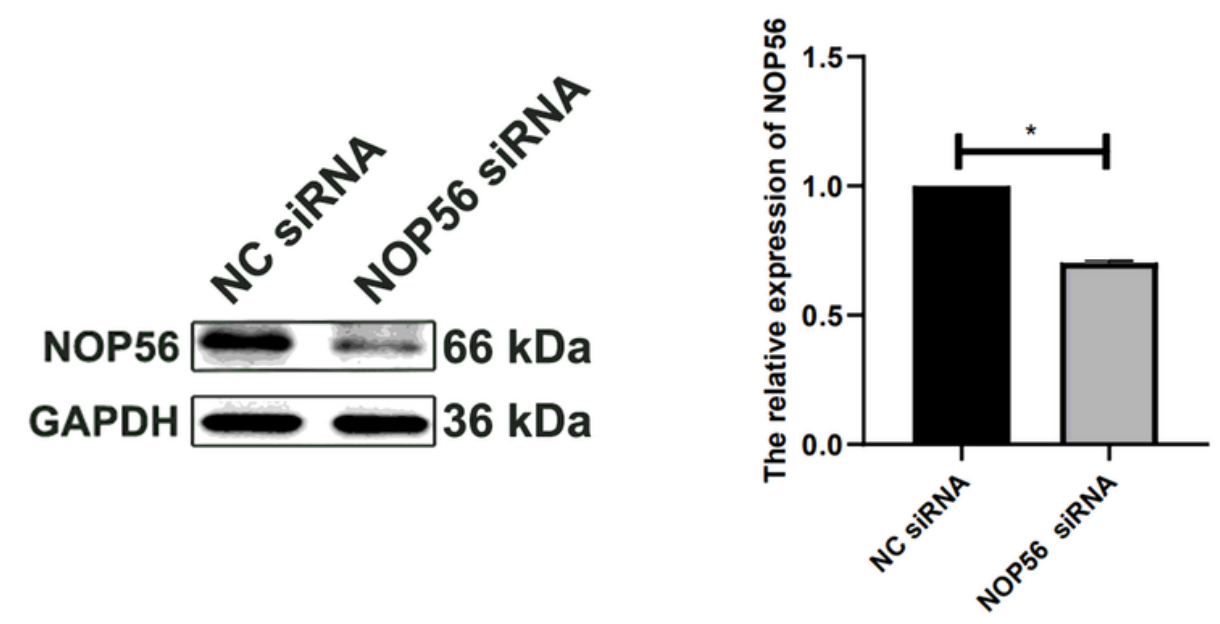

Figure 2

Expression of piRNA MW557525/NOP56 and their successful transfection in Piwil2-iCSCs. A-B: Expression of piRNA MW557525 and NOP56 in Piwil2-iCSCs compared with FBs as shown by qRT-PCR. C: piRNA MW557525 expression was measured via qRT-PCR to confirm mimic and inhibitor transfection efficiency in cells. D-E: NOP56 mRNA level was assayed via qRT-PCR and WB analyses after transfection with NOP56 siRNA. For comparisons, Student's $t$ test was performed; ${ }^{*} P<0.05,{ }^{\star} * \mathrm{P}<0.01,{ }^{\star \star *} \mathrm{P}<0.001$, $\star \star \star \star P<0.0001$. 
A
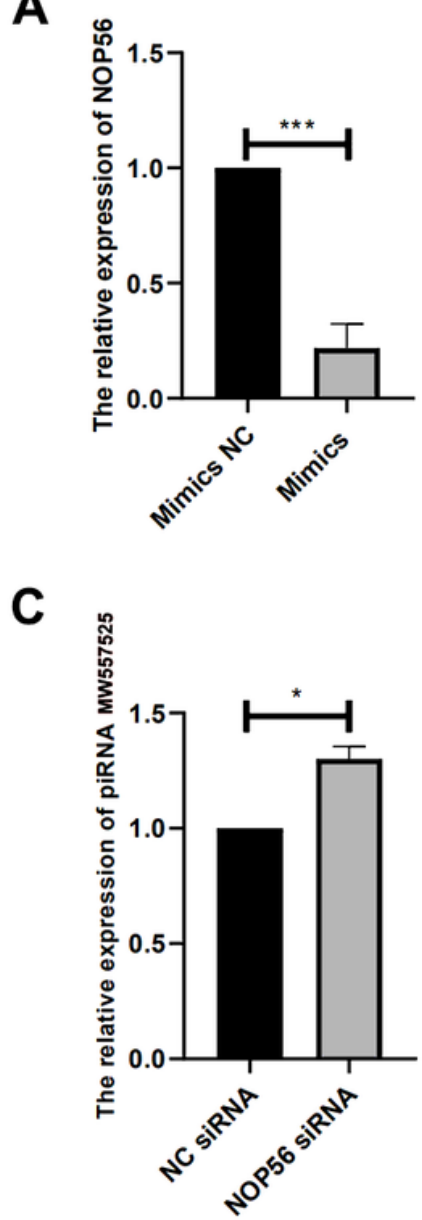

B

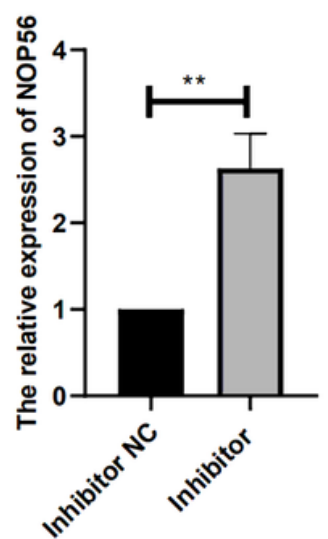

D

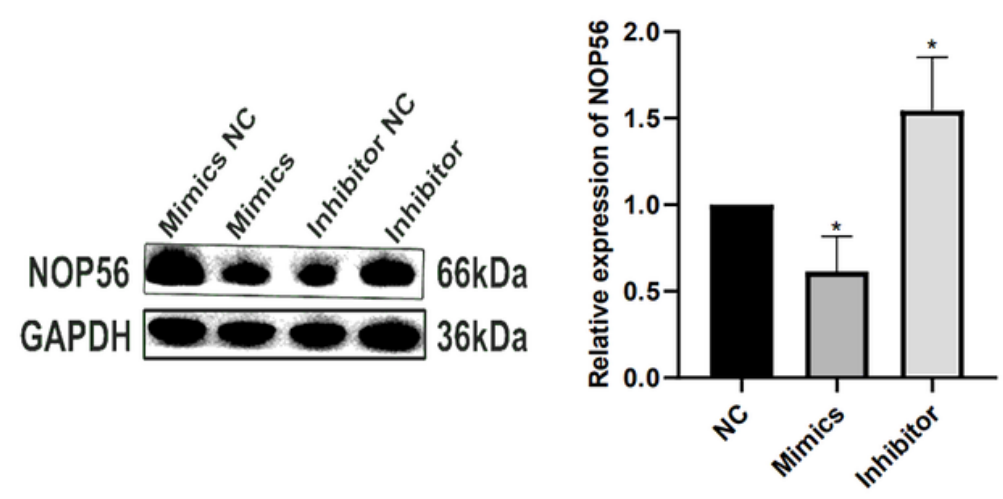

$\mathbf{E}$

\section{Figure 3}

Position 1837-1843 of NOP56 3'UTR

NOP563'UTR: $\quad$ 5'...GGGAGGUGGGGCAUAC MW557525: $\quad$ 3'ACCUCCACUACUUG....A

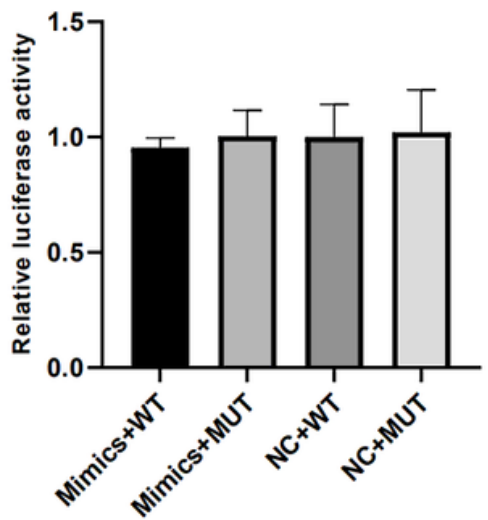

PiRNA MW557525 was negatively associated with NOP56 in Piwil2-iCSCs. A-B: NOP56 mRNA level was assayed via qRT-PCR and WB analyses after cell transfection with piRNA MW557525 mimic, inhibitor or control. C: Effect of NOP56 silencing on piRNA MW557525 expression after cells were transfected with NOP56 siRNA plasmid or empty vector. D:Binding seqeunces between piRNA MW557525 and NOP56 was forecast by Bioinformatics prediction algorithms. E:LRA of cells after cotransfection with WT or MUT NOP56 3'-UTR and piRNA MW557525. For comparisons, Student's $t$ test was performed; ${ }^{*} P<0.05,{ }^{\star * P}<$ $0.01, * \star \star P<0.001$. 
A
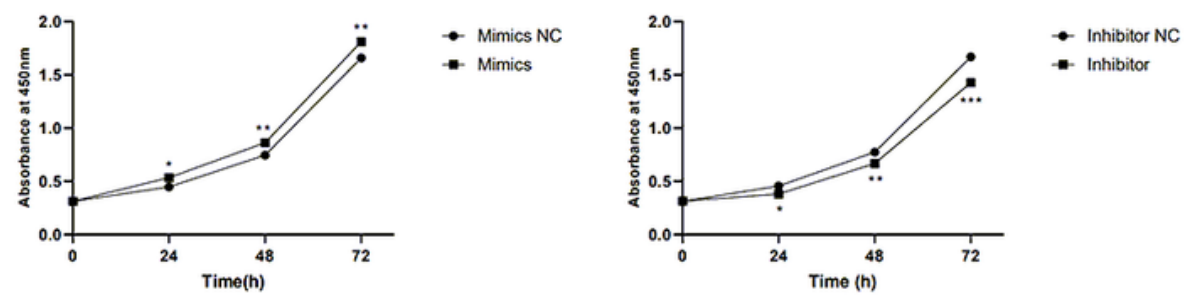

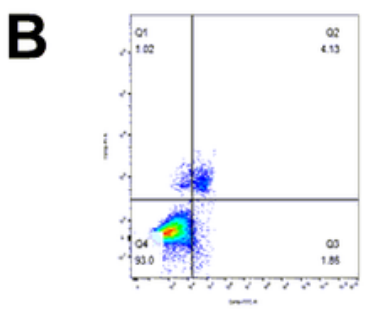

Mimics NC

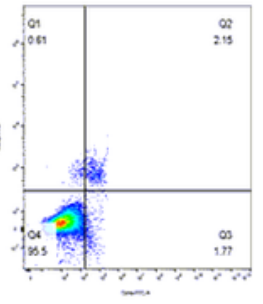

Mimcs

C

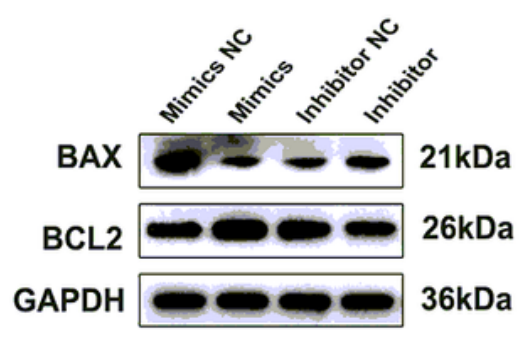

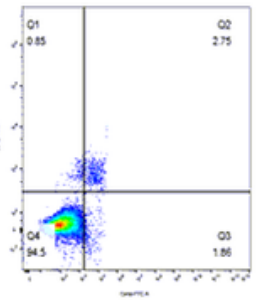

Inhibitor NC

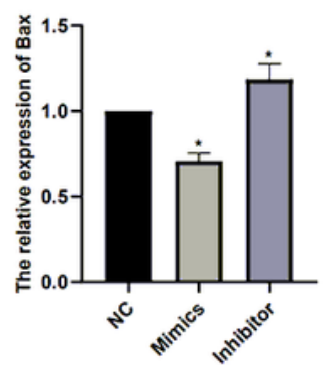

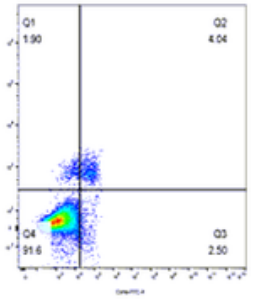

Inhibitor
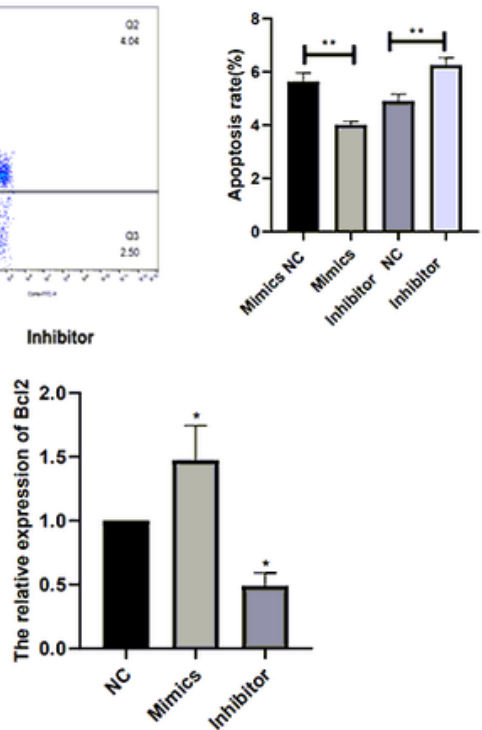

D

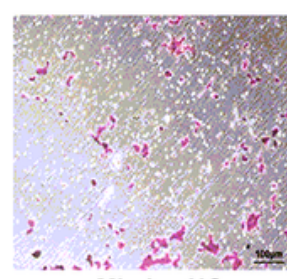

Mimics NC

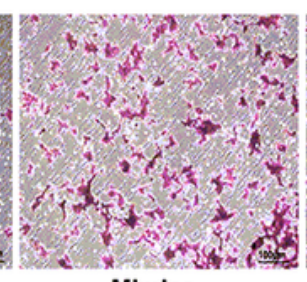

Mimics

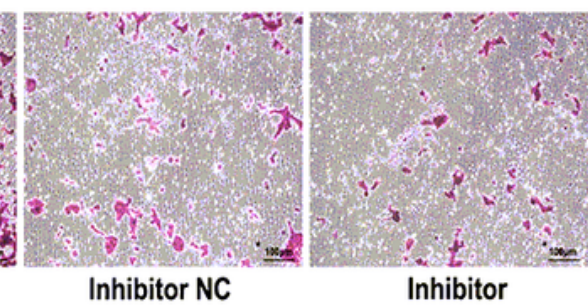

Inhibitor NC

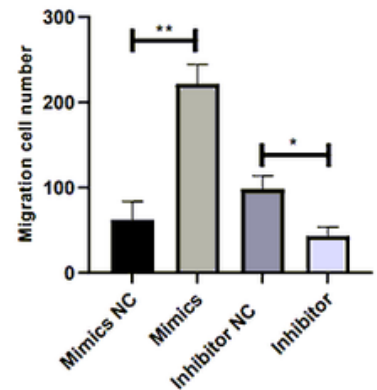

E

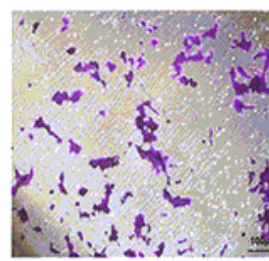

Mimics NC

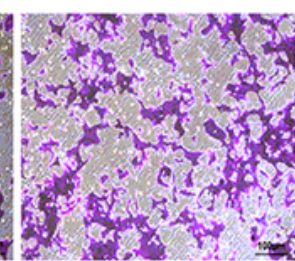

Mimics

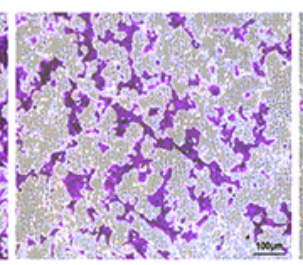

Inhibitor NC

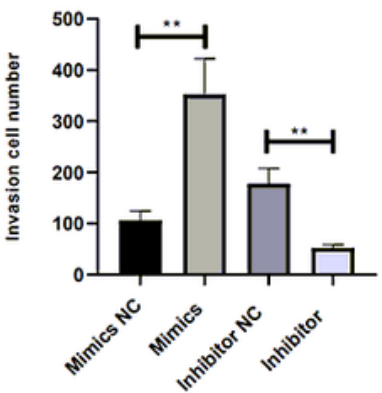

\section{Figure 4}

PiRNA MW557525 promoted cell proliferation, migration and invasion and inhibited apoptosis. Piwil2iCSCs were transfected with piRNA MW557525 mimic, inhibitor or NC. A: We performed a CCK-8 assay to determine cell proliferation. B-C: Cell apoptosis was detected by FCM and WB assays. D-E: Transwell assays were performed to assess migration and invasion of Piwil2-iCSCs. Results are shown as the mean \pm SD. For comparisons, Student's t test was performed; ${ }^{*} P<0.05,{ }^{*} \mathrm{P}<0.01, * \star \star P<0.001$ vs. NC. 
A

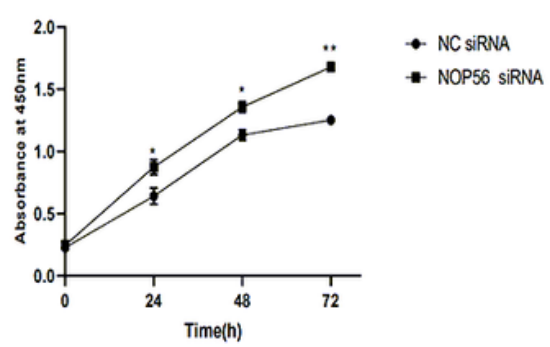

C

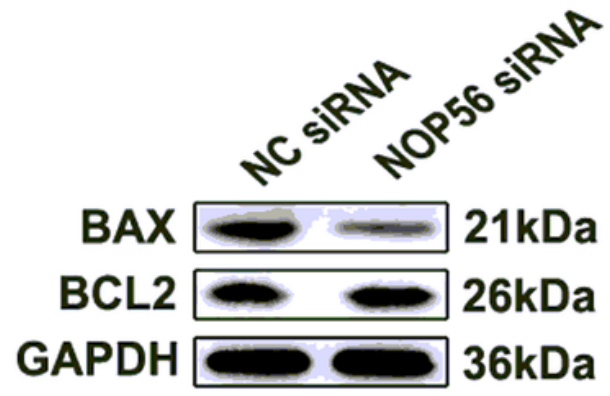

B

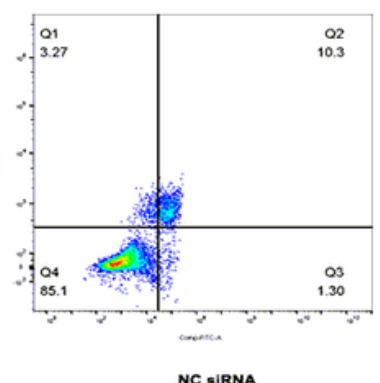

NC SIRNA
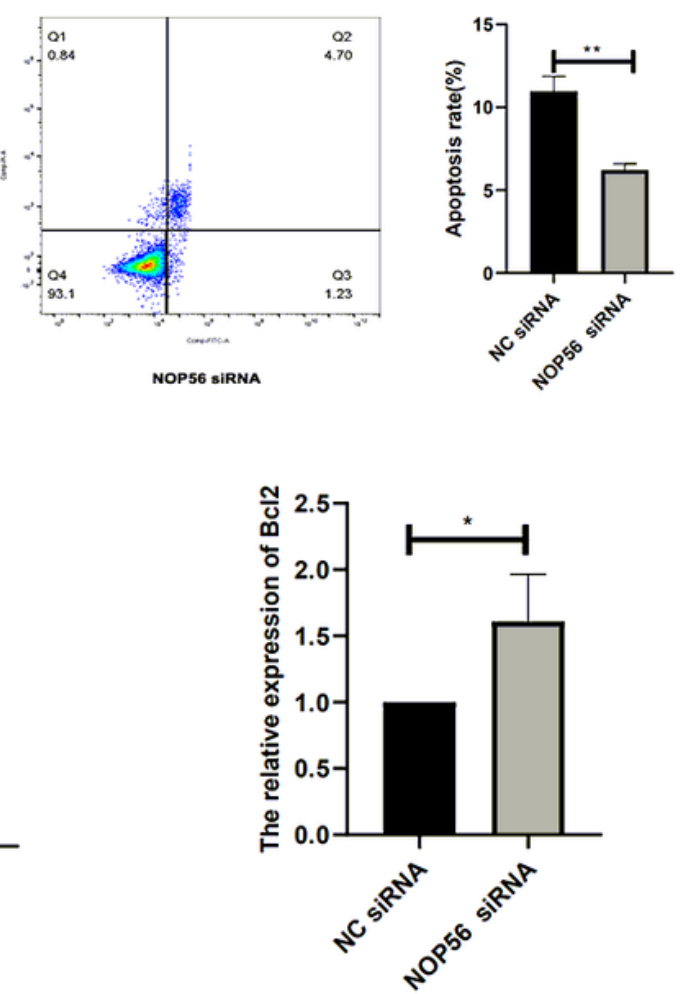

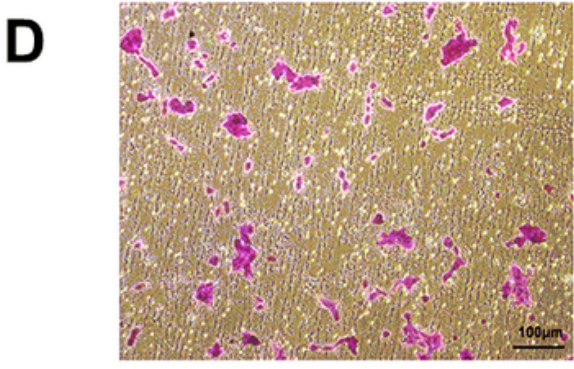

NC siRNA

$\mathbf{E}$

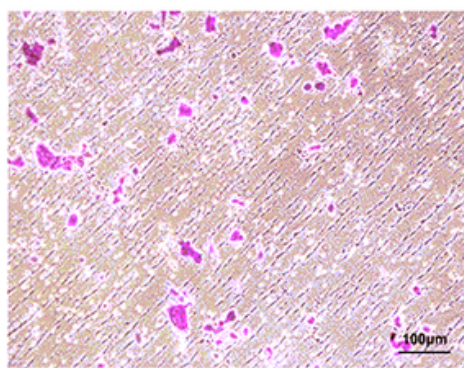

NC SIRNA

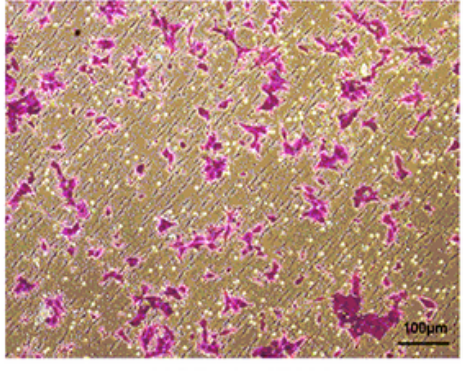

NOP56 siRNA

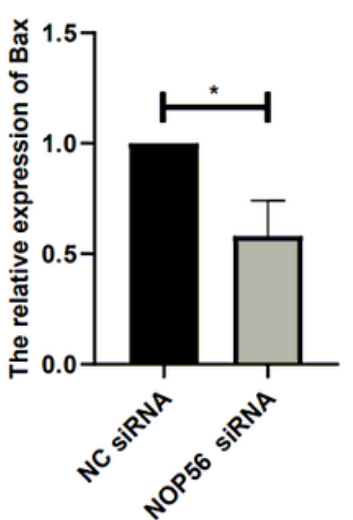

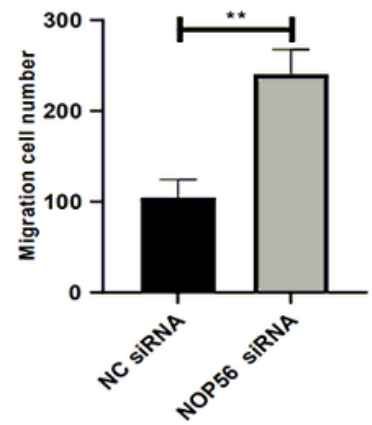

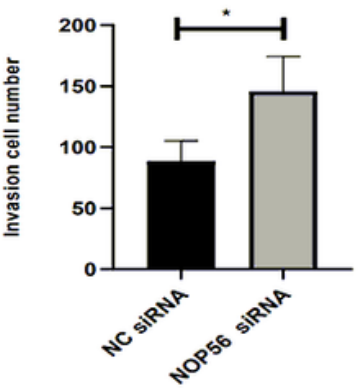

Figure 5

NOP56 knockdown promoted cell proliferation, migration and invasion and suppressed apoptosis. Piwil2iCSCs were transfected with NOP56 siRNA or NC siRNA. A: Cell proliferation was assessed at the indicated time points via CCK-8 assay. B-C: FCM and WB analyses were performed to determine cell apoptosis. D-E: A transwell assay was performed to evaluate cell migration and invasion in vitro. Results are shown as the mean $\pm S D$. For comparisons, Student's $t$ test was performed; ${ }^{*} P<0.05, * \star P<0.01, \star \star \star P$ $<0.001$ vs. NC. 
A
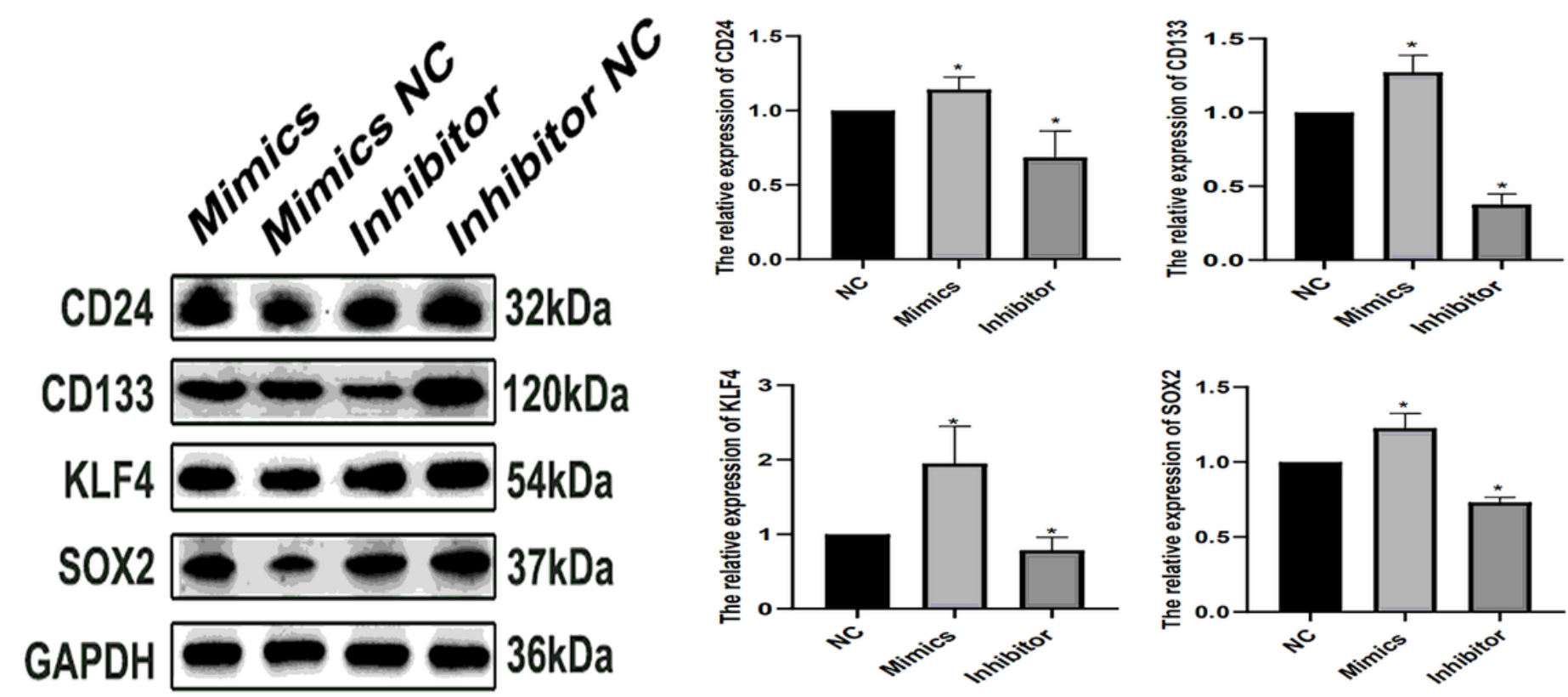

B
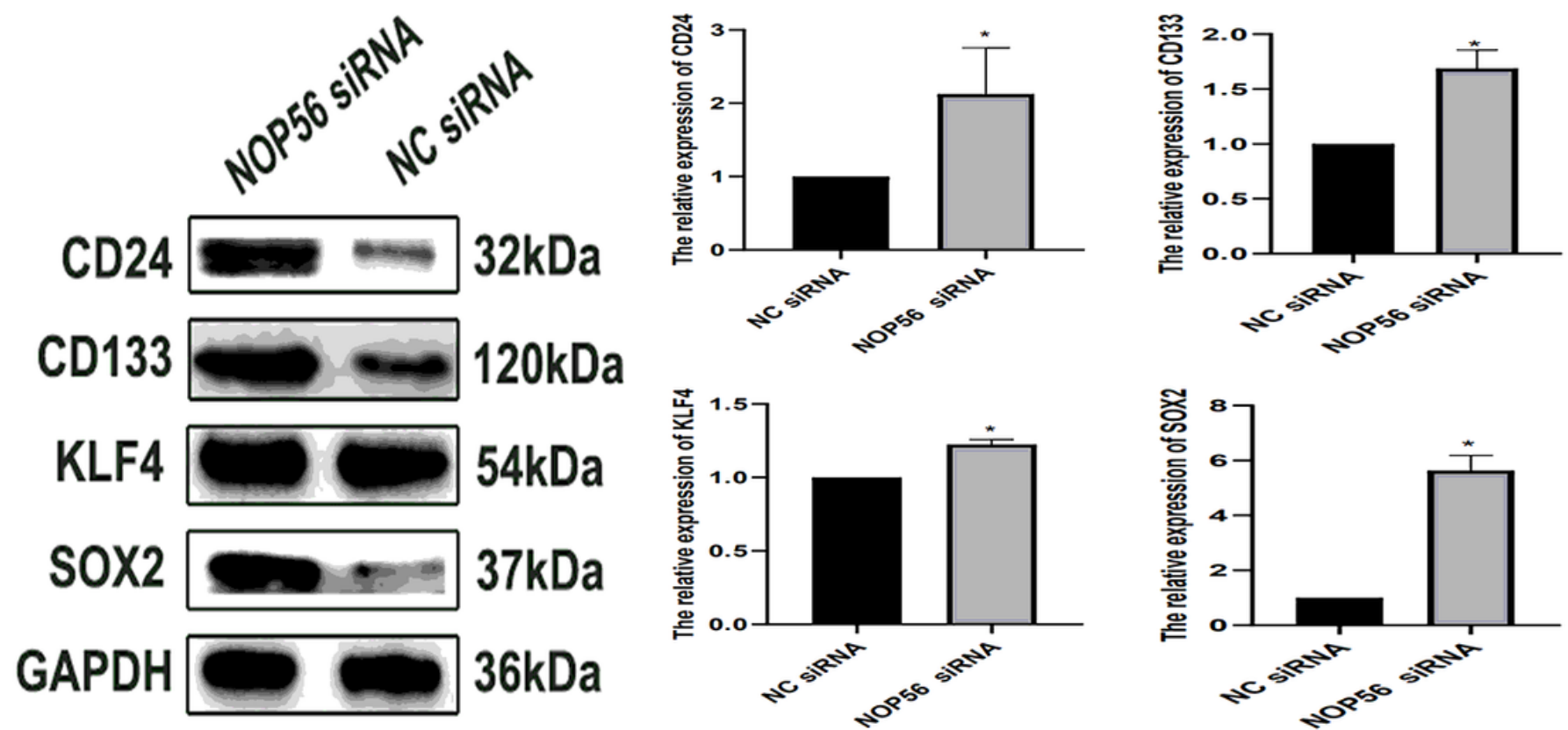

Figure 6

piRNA MW557525 and NOP56 regulated the pluripotency of Piwil2-iCSCs. A: CD24, CD133, KLF4, SOX2 and GAPDH levels were detected via WB $72 \mathrm{~h}$ after transfection with piRNA MW557525 mimics, inhibitor, or NC.B: Relative expression of CD24, CD133, KLF4, SOX2 and GAPDH was detected via WB $72 \mathrm{~h}$ after cell transfection with NOP56-specific siRNAs. Columns represent the mean of three independent experiments and the bars represent $S D ; * P<0.05$. 